Louisiana State University

LSU Digital Commons

Faculty Publications

Department of Mathematics

$1-1-2019$

\title{
A chain theorem for 4-connected graphs
}

Chengfu Qin

Guangxi Teachers Education University

Guoli Ding

Louisiana State University

Follow this and additional works at: https://digitalcommons.Isu.edu/mathematics_pubs

\section{Recommended Citation}

Qin, C., \& Ding, G. (2019). A chain theorem for 4-connected graphs. Journal of Combinatorial Theory. Series B, 134, 341-349. https://doi.org/10.1016/j.jctb.2018.07.005

This Article is brought to you for free and open access by the Department of Mathematics at LSU Digital Commons. It has been accepted for inclusion in Faculty Publications by an authorized administrator of LSU Digital Commons. For more information, please contact ir@lsu.edu. 


\title{
A chain theorem for 4-connected graphs
}

\author{
Chengfu Qin ${ }^{a}$, Guoli Ding ${ }^{b}$ \\ a Department of Mathematics, Guangxi Teachers Education University, Nanning, \\ China \\ b Department of Mathematics, Louisiana State University, Baton Rouge, USA
}

\section{A R T I C L E I N F O}

\section{Article history:}

Received 26 June 2015

Available online 14 July 2018

\section{Keywords:}

4-connected graphs

Chain theorem

Contractible edge

Nonplanar graphs

\author{
A B S T R A C T
}

A sequence of 4-connected graphs $G_{0}, G_{1}, \ldots, G_{n}$ is called a $\left(G_{0}, G_{n}\right)$-chain if each $G_{i}(i<n)$ has an edge $e_{i}$ such that $G_{i} / e_{i}=G_{i+1}$. A classical result of Martinov states that for every 4-connected graph $G$ there exists a $(G, H)$-chain such that $H \in \mathcal{C} \cup \mathcal{L}$, where $\mathcal{C}=\left\{C_{n}^{2}: n \geq 5\right\}$ and $\mathcal{L}=\{L:$ $L$ be the line graph of a cyclically 4-edge-connected cubic graph $\}$. This result is strengthened in this paper as follows. Suppose $G$ is 4-connected and $G \notin \mathcal{C} \cup \mathcal{L}$. Then there exists a $\left(G, C_{6}^{2}\right)$-chain if $G$ is planar and a $\left(G, C_{5}^{2}\right)$-chain if $G$ is nonplanar.

๑) 2018 Elsevier Inc. All rights reserved.

\section{Introduction}

The purpose of this paper is to improve a well-known classical chain theorem of Martinov [10] for 4-connected graphs. We begin by formally stating this result.

All graphs considered in this paper are simple. In particular, $G / e$ denotes the graph obtained from $G$ by contracting an edge $e$ and then deleting parallel edges. For each integer $n \geq 5$, let $C_{n}^{2}$ be the graph obtained from an $n$-cycle $C_{n}$ by joining vertices of distance two in the cycle. Notice that $C_{5}^{2}$ is $K_{5}$ and $C_{6}^{2}$ is the octahedron. In general, $C_{n}^{2}$ is 4-connected, and it is planar if and only if $n$ is even. Let $\mathcal{C}=\left\{C_{n}^{2}: n \geq 5\right\}$.

E-mail address: qtclf@163.com (C. Qin). 
A graph $G$ with at least six vertices is called cyclically $k$-edge-connected if the deletion of fewer than $k$ edges from $G$ does not create two components which both contain at least one cycle. We remark that all cyclically 4-edge-connected cubic graphs can be constructed from either $K_{3,3}$ or the cube by repeatedly applying an operation known as "adding a handle" $[3-6,12]$. Let $\mathcal{L}=\{L: L$ be the line graph of an cyclically 4-edge-connected cubic graph\}. The following is the chain theorem of Martinov [10] (a similar result using both edge contractions and deletions was given by Fontet [3]).

Theorem 1.1. For every 4-connected graph $G$ there exists a sequence of 4-connected graphs $G_{0}, G_{1}, \ldots, G_{n}$ such that $G_{0}=G, G_{n} \in \mathcal{C} \cup \mathcal{L}$, and every $G_{i}(i<n)$ has an edge $e_{i}$ for which $G_{i} / e_{i}=G_{i+1}$.

This result provides a very useful tool for analyzing 4-connected graphs. Under the current setting, it says that every 4-connected graph $G$ can be reduced, within the class of 4-connected graphs, to a graph $G_{n} \in \mathcal{C} \cup \mathcal{L}$ by repeatedly contracting edges. If we reverse this process then the theorem tells us that every desired (usually unknown) 4-connected graph $G$ can be constructed from a graph $G_{n} \in \mathcal{C} \cup \mathcal{L}$ by repeated "uncontractions". This approach is used successfully in characterizing 4-connected graphs that do not contain a minor isomorphic to the cube [8], to the octahedron [7], to the octahedron plus an edge [9], or to the complement of $P_{7}$ [2]. However, this theorem has two major defects which limit its further applications.

First, for a general 4-connected graph $G$, the starting graph $G_{n}$ in the construction sequence could be any graph in $\mathcal{C} \cup \mathcal{L}$. What this means is that, in order to obtain $G$ we have to consider infinitely many possible choices for $G_{n}$, and this increases the complexity of our analysis. It would be nice if we can narrow down the choices for $G_{n}$. The second defect of the theorem, which causes an even bigger problem, is that $G_{n}$ could be planar even if $G$ is nonplanar. As a consequence, in order to construct $G$, we have to examine many planar graphs, which often are useless for constructing $G$. The following is the main result of this paper, which addresses both concerns. Let us call a sequence as described in Theorem 1.1 a $\left(G, G_{n}\right)$-chain.

Theorem 1.2. Let $G$ be a 4-connected graph not in $\mathcal{C} \cup \mathcal{L}$. If $G$ is planar then there exists $a\left(G, C_{6}^{2}\right)$-chain; if $G$ is nonplanar then there exists a $\left(G, K_{5}\right)$-chain.

As an application, we prove the following main result of [9]. Let $\mathrm{Oct}^{+}$denote the unique graph obtained from the octahedron by adding an edge.

Theorem 1.3. If a 4-connected nonplanar graph $G$ has no Oct ${ }^{+}$-minor then $G=C_{2 n+1}^{2}$ for some $n \geq 2$.

Proof. Suppose the result is false. By Theorem 1.2, either there exists a $\left(G, K_{5}\right)$-chain or $G=L(H)$ for a nonplanar cubic graph $H$. The second case is impossible since $L(H)$ contains $L\left(K_{3,3}\right)$, which contains $O c t^{+}$. The first case is impossible either because $G$ has 
to contain one of the three 4-connected uncontractions of $K_{5}$, which are $K_{6}, K_{6} \backslash e, O c t^{+}$, yet all of them contain $\mathrm{Oct}^{+}$.

We close this section by introducing a few definitions. For any graph $G$, let $V(G)$ and $E(G)$ denote the vertex set and edge set of $G$, respectively. If $X \subseteq V(G)$, let $N_{G}(X)=\{y \in V(G)-X: y x \in E(G)$ for some $x \in X\}$. Members of $N_{G}(X)$ are neighbors of $X$ and the set $N_{G}(X)$ is the neighborhood of $X$. For any $x \in V(G)$, we will write $N_{G}(x)$ for $N_{G}(\{x\})$. As usual, $\left|N_{G}(x)\right|$ is the degree of $x$, which is denoted by $d_{G}(x)$. Let $E_{G}(x)$ stand for the set of edges of $G$ that are incident with $x$. We will drop the subscript $G$ if there is no need to emphasize $G$.

Let $G=(V, E)$ be a graph. A set $T \subseteq V$ is called a $k$-separator of $G$ if $|T|=k$ and $G-T$ is disconnected. As usual, $G$ is $k$-connected if $|V|>k$ and $G$ has no $k^{\prime}$-separator with $k^{\prime}<k$. Let $G$ be a $k$-connected graph. An edge $e$ of $G$ is said to be $k$-contractible if $G / e$ is again $k$-connected. We may simply call $e$ contractible if $k$ is clear from the context. The new vertex of $G / e$ will be denoted by $\bar{e}$. Observe that an edge $x y$ of a non-complete graph $G$ is not $k$-contractible if and only if $G$ has a $k$-separator containing both $x$ and $y$.

Let $T$ be a $k$-separator of a $k$-connected graph $G$. A $T$-fragment of $G$ is the vertex set of a union of at least one but not all components of $G-T$. We often leave out the prefix $T$ when we do not need to emphasize it. If $A$ is a fragment of $G$ then it is clear that $N(A)$ is a $k$-separator. Let us define $\bar{A}=V(G)-A-N(A)$. Then $\bar{A}$ is also a fragment of $G$ with $N(A)=N(\bar{A})$. Notice that, for any $x \in A, x$ has no neighbors in $\bar{A}$.

The organization of this paper is as follows. In Section 2, we establish a few lemmas on contractible edges. Then, in Section 3, we prove our key lemma. Finally, we prove Theorem 1.2 in Section 4.

\section{Contractible edges}

In this section we present a few lemmas on contractible edges. We first establish that every 4 -connected graph not in $\mathcal{C} \cup \mathcal{L}$ can be reduced to $K_{5}$ or the octahedron. Our proof is divided into two steps.

Lemma 2.1. Suppose a $k$-connected $(k \geq 2)$ graph $G$ has a contractible edge $e=x y$ such that $d_{G / e}(\bar{e})=k$ and the neighborhood of $\bar{e}$ does not contain $K_{2, k-2}$ as a subgraph. Then $G$ has an edge $e^{\prime}$ such that $G / e^{\prime}$ is isomorphic to a graph obtained from $G / e$ by adding at least one extra edge.

Proof. Let $N_{G / e}(\bar{e})=\left\{z_{1}, z_{2}, \ldots, z_{k}\right\}=Z$. Then $N_{G}(x) \subseteq Z \cup\{y\}$ and $N_{G}(y) \subseteq Z \cup\{x\}$. Since $d_{G}(x) \geq k$ and $d_{G}(y) \geq k$, we may assume, by adjusting the indices if necessary, that $N_{G}(x) \supseteq Z-\left\{z_{1}\right\}$ and $N_{G}(y) \supseteq Z-\left\{z_{2}\right\}$. By considering $G / e$ and $G / x z_{2}$ as graphs obtained by adding edges to $G-x$, we observe that $G / x z_{2}$ is isomorphic to the graph obtained from $G / e$ by adding edges $z_{2} z_{3}, z_{2} z_{4}, \ldots, z_{2} z_{k}$ (and also possibly $z_{2} z_{1}$ ). If $e^{\prime}=x z_{2}$ does not satisfy the lemma, then $z_{2} z_{3}, z_{2} z_{4}, \ldots, z_{2} z_{k}$ are all edges of $G / e$. This 
implies that $z_{1} z_{3}, z_{1} z_{4}, \ldots, z_{1} z_{k}$ are not all edges of $G / e$ and thus $e^{\prime}=y z_{1}$ satisfies the lemma.

Corollary 2.2. Suppose $e$ is an edge of a 4-connected graph $G$ such that $G / e \in \mathcal{C} \cup \mathcal{L}$. Then, unless $G / e=C_{5}^{2}$ or $C_{6}^{2}, G$ has an edge $e^{\prime}$ such that $G / e^{\prime}$ is isomorphic to a graph obtained from $G /$ e by adding at least one extra edge.

Proof. If $G / e=L(H)$, where $H$ is an cyclically 4-edge-connected cubic graph, then the neighborhood of $\bar{e}$ induces a matching since $H$ is triangle free. Thus the result holds by Lemma 2.1. If $G / e=C_{n}^{2}$ for some $n \geq 7$, then the neighborhood of $\bar{e}$ induces a path and, again, the result holds by Lemma 2.1 .

We also need the next three lemmas.

Lemma 2.3 ([1]). If $x$ is a vertex of a 4-connected graph $G$ with $d(x) \geq 5$, then $G$ has a contractible edge contained in $E(y)$ for some $y \in N(x)$.

Lemma 2.4. Let $x y$ and $x z$ be two edges of a $k$-connected graph $G$ with $N(x) \subseteq N(y) \cup$ $\{y, z\}$. If $x y$ is $k$-contractible then so is $x z$.

Proof. Suppose $x z$ is non-contractible. Then $G$ has a $k$-separator $T$ containing both $x$ and $z$. Notice that $y \notin T$ since $x y$ is contractible. Let $A$ be a $T$-fragment that contains $y$. Now, since $N(x) \subseteq N(y) \cup\{y, z\}$, we find that $x$ has no neighbor in $\bar{A}$, contradicting the $k$-connectivity of $G$.

Lemma 2.5. Let $e=x y$ be a $k$-contractible edge of a $k$-connected graph $G$. Let $e^{\prime}$ be an edge of $G / e$ (and hence also an edge of $G$ ). If $e^{\prime}$ is $k$-contractible in $G / e$ but not in $G$, then some $z \in\{x, y\}$ has degree $k$ and is such that $N_{G}(z)$ contains both ends of $e^{\prime}$.

Proof. Let $x^{\prime}, y^{\prime}$ be the two ends of $e^{\prime}$ in $G$. Since $e^{\prime}$ is not contractible in $G, G$ has a $k$-separator $T$ that contains both $x^{\prime}$ and $y^{\prime}$. Clearly, $\{x, y\}-T \neq \emptyset$ since $e$ is contractible. Let $A$ be a $T$-fragment with $A \cap\{x, y\} \neq \emptyset$. By symmetry, we may assume $x \in A$. If $y \in A$ then $T$ is a $k$-separator of $G / e$ with $T \supseteq\left\{x^{\prime}, y^{\prime}\right\}$, contradicting the contractibility of $e^{\prime}$. So we must have $y \in T$. If $|A| \geq 2$ then $T^{\prime}=(T-\{y\}) \cup\{\bar{e}\}$ is a $k$-separator of $G / e$ and $T^{\prime}$ contains both ends of $e^{\prime}$ in $G / e$. This is again a contradiction. It follows that $A=\{x\}$ and thus the Lemma holds with $z=x$.

\section{A key lemma}

Let $x, y, z$ be three distinct vertices of a cycle $C$. Then $C$ has two subpaths with ends $x$ and $y$. We denote the vertex set of the path that contains $z$ by $C[x, z, y]$, and we denote the vertex set of the other path by $C[x, \bar{z}, y]$. We also define $C(x, z, y)=C[x, z, y]-\{x, y\}$, $C(x, z, y]=C[x, z, y]-\{x\}$, and $C[x, z, y)=C[x, z, y]-\{y\}$. In addition, $C(x, \bar{z}, y)$, 
$C(x, \bar{z}, y]$, and $C[x, \bar{z}, y)$ are defined analogously. The purpose of this section is to prove the following.

Lemma 3.1. If a 4-connected nonplanar graph $G$ does not belong to $\mathcal{C} \cup \mathcal{L}$, then $G$ has an edge e such that $G /$ e remains 4 -connected and nonplanar.

Proof. It is hard to separate our proof into independent lemmas, so this proof will last till the end of this section. To make the proof easier to follow, we divide it into a sequence of claims.

By Theorem 1.1, $G$ has at least one contractible edge. We assume that, for every contractible edge $e$ of $G, G / e$ is planar because otherwise we are done. Let $e=x y$ be a contractible edge of $G$. It follows that $G / e$ is planar. This implies $|V(G)| \geq 7$ because otherwise $G / e$ would be a 4 -connected planar graph on at most five vertices, which is impossible.

Let us consider the unique planar embedding of $G / e$. This embedding induces an embedding of $(G / e)-\bar{e}$. Notice that this embedding of $(G / e)-\bar{e}$ has a face $F$ such that, in the planar embedding of $G / e$, all edges of $E_{G / e}(\bar{e})$ are embedded in $F$. Since $G / e$ is 4-connected, $(G / e)-\bar{e}$ is 3-connected, which implies that $F$ is bounded by a cycle $C$ of $(G / e)-\bar{e}$ and this cycle contains all neighbors of $\bar{e}$. Let $B=G-(V(C) \cup\{x, y\})$. It is easy to see that, as a facial cycle of the 3-connected planar graph $G-x-y=(G / e)-\bar{e}, C$ satisfies the following, which, using the language of Tutte [11], says that $C$ is peripheral in $G-x-y$.

Claim 1. $C$ is an induced cycle of $G, B$ is connected, and $N(V(B))=V(C)$.

Let $x_{1}, x_{2}, \cdots, x_{s}$ be the neighbors of $x$ (other than $y$ ), which are listed in the order they appear on $C$. Let $N(y)=\left\{x, y_{1}, y_{2}, \cdots, y_{t}\right\}$. For the purpose of simplifying our notation, we do not require $y_{1}, y_{2}, \ldots, y_{t}$ to be listed in a specific order. This setting creates a non-symmetry between $x$ and $y$. As a result, in the following discussions, some of our statements are only made for one of $x, y$. We point out that these statements are still valid if we swap $x$ and $y$, since $x$ and $y$ are indeed symmetric.

A quadruple $\left(x_{i}, y_{j}, x_{k}, y_{l}\right)$ is said to be crossing if the four vertices are distinct and $y_{j}, y_{l}$ are contained in different components of $C-\left\{x_{i}, x_{k}\right\}$.

Claim 2. There exists a crossing quadruple.

Proof. Suppose $\left\{x_{1}, x_{2}, \ldots, x_{s}\right\}=\left\{y_{1}, y_{2}, \ldots, y_{t}\right\}$. Since $G / e$ is 4 -connected, we must have $s \geq 4$ and thus Claim 2 follows. Next, we assume by symmetry that $y_{1} \notin\left\{x_{1}, x_{2}, \ldots, x_{s}\right\}$. Choose $i$ such that $C\left(x_{i}, y_{1}, x_{i+1}\right)$ contains no neighbors of $x$ (in this section the indices on the letter $x$ are always taken modulo $s)$. Since $G$ is nonplanar, $C\left(x_{i}, \bar{y}_{1}, x_{i+1}\right)$ must contain a neighbor of $y$ and thus Claim 2 is proved. 
When we say " $y_{j}$ is contained in a crossing quadruple" we mean that there exists a crossing quadruple of the form $\left(x_{i}, y_{j}, x_{k}, y_{l}\right)$. We need to make this clear since in general $y_{j}$ could be equal to some $x_{i}$.

Claim 3. Every $y_{j}$ is contained in a crossing quadruple, unless $y_{j}=x_{r}$ for some $r$ and $N(y)-\{x\} \subseteq C\left[x_{r-1}, x_{r}, x_{r+1}\right]$. Moreover, there is at most one $y_{j}$ that is not contained in any crossing quadruple.

Proof. By Claim 2, $G$ has crossing quadruple $\left(x_{m}, y_{a}, x_{n}, y_{b}\right)$. If $y_{j} \notin\left\{x_{m}, x_{n}\right\}$ then either $\left(x_{m}, y_{a}, x_{n}, y_{j}\right)$ or $\left(x_{m}, y_{j}, x_{n}, y_{b}\right)$ is crossing. So we may assume $y_{j}=x_{m}$. If $C\left(x_{m-1}, \bar{x}_{m}, x_{m+1}\right)$ contains a neighbor $y_{l}$ of $y$, then $\left(x_{m-1}, y_{j}, x_{m+1}, y_{l}\right)$ is a crossing quadruple. Else $r=m$ satisfies the lemma. Finally, if in addition to $y_{j}$, vertex $y_{j^{\prime}}\left(j^{\prime} \neq j\right)$ is not contained in any crossing quadruple either, then the first part of the lemma implies either $y_{j^{\prime}}=x_{r-1}$ and $N(y)-\{x\} \subseteq C\left[x_{r-2}, x_{r-1}, x_{r}\right]$ or $y_{j^{\prime}}=x_{r+1}$ and $N(y)-$ $\{x\} \subseteq C\left[x_{r}, x_{r+1}, x_{r+2}\right]$, which in turns implies either $N(y)-\{x\} \subseteq C\left[x_{r-1}, \bar{x}_{r+1}, x_{r}\right]$ or $N(y)-\{x\} \subseteq C\left[x_{r}, \bar{x}_{r-1}, x_{r+1}\right]$, contradicting the non-planarity of $G$.

Claim 4. If $\left(x_{i}, y_{j}, x_{k}, y_{l}\right)$ is a crossing quadruple, then $G / y y_{j}$ is nonplanar.

Proof. It is clear that $G / y y_{j}-V(B)$ has a $K_{4}$ minor whose four branch sets each contain exactly one member of $\left\{x_{i}, \overline{y y_{j}}, x_{k}, y_{l}\right\}$. By Claim $1, B$ is connected and each vertex of $C$ has a neighbor in $B$. Thus $G / y y_{j}$ has a $K_{5}$ minor, which proves that $G / y y_{j}$ is nonplanar.

Since we have assumed that $G / e$ is planar if $e$ is contractible, we deduce the following from Claim 4.

Claim 5. If $\left(x_{i}, y_{j}, x_{k}, y_{l}\right)$ is a crossing quadruple, then $y y_{j}$ is not contractible.

Claim 6. Suppose $T$ is a 4-separator of $G$ that contains both $y$ and some $y_{j}$. Then either $T=N(x)$ or $T=\left\{y, y_{j}, z, z^{\prime}\right\}$ for some $z \in V(C)-\left\{y_{j}\right\}$ and $z^{\prime} \in V(B)$.

Proof. It is clear that $x \notin T$ since $x y$ is contractible in $G$. Let $A$ be a $T$-fragment of $G$ with $x \in A$. If $N(x)=T$ then we are done. So let $x$ have a neighbor $x^{*} \in A$. Clearly, $x^{*} \in V(C)$ as $x^{*} \neq y$. Since $y \in T, y$ must have a neighbor $y^{*} \in \bar{A}$. Observe that $y^{*} \in V(C)$ as $y^{*} \neq x$. Therefore, as $T$ separates $x^{*}$ from $y^{*}, T$ must contain a vertex $z \in C\left(x^{*}, \bar{y}_{j}, y^{*}\right) \subseteq V(C)-\left\{y_{j}\right\}$. On the other hand, since $x^{*}, y^{*} \in V(C)$, we deduce from Claim 1 that $G$ has a path $P$ between $x^{*}, y^{*}$ such that $V(P)-\left\{x^{*}, y^{*}\right\} \subseteq V(B)$. Again, since $T$ separates $x^{*}$ from $y^{*}, T$ must contain a vertex $z^{\prime} \in V(P)-\left\{x^{*}, y^{*}\right\} \subseteq$ $V(B)$. 
Claim 7. Suppose $T$ is a 4-separator of $G$ such that $T=\left\{y, y_{j}, z, z^{\prime}\right\}$ for some $z \in$ $V(C)-\left\{y_{j}\right\}$ and $z^{\prime} \in V(B)$. Then for any distinct $x_{i}, x_{k}$ different from $y_{j}, C\left(x_{i}, y_{j}, x_{k}\right)$ contains at least two neighbors of $y$.

Proof. Let $A$ be a $T$-fragment of $G$ with $x \in A$. Let $P_{1}, P_{2}$ be the two subpaths of $C$ with ends $y_{j}$ and $z$. Since $T \cap V(C)=\left\{y_{j}, z\right\}$, for $i=1,2, V_{i}=V\left(P_{i}\right)-\left\{y_{j}, z\right\}$ is entirely contained in $A$ or $\bar{A}$. Notice that $x$ has a neighbor in $A$ since $d(x) \geq 4$ and $x z^{\prime} \notin E(G)$. It follows that $V(C) \cap A \neq \emptyset$. On the other hand, $V(C) \cap \bar{A} \neq \emptyset$ since $y$ has a neighbor $y_{l}$ in $\bar{A}$. Hence, we may assume $V_{1} \subseteq A$ and $V_{2} \subseteq \bar{A}$. Observe that $N(x)-\{y\} \subseteq V\left(P_{1}\right)$, it follows that $C\left(x_{i}, y_{j}, x_{k}\right)$ contains $y_{j}$ and $y_{l}$ for any distinct $x_{i}, x_{k}$ different from $y_{j}$. Hence Claim 7 holds.

Claim 8. $|N(\{x, y\})| \geq 5$.

Proof. Suppose $|N(\{x, y\})| \leq 4$. Then $|N(\{x, y\})|=4$ since $G$ is 4-connected with $|V(G)| \geq 7$. Choose $z \in N(\{x, y\})$ such that, if possible, $z$ is adjacent to only one of $x, y$. Without loss of generality, we assume $z$ is adjacent to $y$ and thus $z=y_{j}$ for some $j$. Note that $|N(y)-N(x)-\{x\}| \leq 1$ since $|N(\{x, y\})|=4 \leq d(x)$. Then our choice of $z$ implies $N(y) \subseteq N(x) \cup\{x, z\}$ and thus, by Lemma 2.4, yy $y_{j}$ is contractible. Consequently, by Claim $5, y_{j}$ is not contained in any crossing quadruple. By Claim 3 , we must have $y_{j} \in N(x)$. Now the way we choose $z$ implies $N(x)-\{y\}=N(y)-\{x\}$ and in this case $y_{j}$ is clearly contained in a crossing quadruple. This contradiction proves Claim 8 .

Claim 9. Both $d(x) \geq 5$ and $d(y) \geq 5$ hold.

Proof. By Claim 2, $G$ has a crossing quadruple $\left(x_{i}, y_{j}, x_{k}, y_{l}\right)$. Suppose Claim 9 is false. Then we may assume by the symmetry between $x$ and $y$ that either $d(x)>d(y)=4$ holds or $d(x)=d(y)=4$ with $\left|\left\{y_{j}, y_{l}\right\}-N(x)\right| \geq\left|\left\{x_{i}, x_{k}\right\}-N(y)\right|$ holds. Since $d(y)=4$ and thus $|N(y) \cap V(C)|=3$, we may further assume that $y_{j}$ is the only neighbor of $y$ contained in $C\left(x_{i}, y_{j}, x_{k}\right)$.

By Claim 5, $G$ has a 4 -separator $T$ containing both $y$ and $y_{j}$. Note that $T \neq N(x)$ because otherwise $y_{j} \in N(x)$, implying $1 \geq\left|\left\{y_{j}, y_{l}\right\}-N(x)\right| \geq\left|\left\{x_{i}, x_{k}\right\}-N(y)\right|$, and thus $\left\{x_{i}, x_{k}\right\} \cap N(y) \neq \emptyset$. From these observation and $d(x)=|T|=4=d(y)$ we deduce that $N(\{x, y\})=\left\{x_{i}, y_{j}, x_{k}, y_{l}\right\}$, which contradicts Claim 8. Therefore, by Claim 6 and Claim $7, y$ has at least two neighbors in $C\left(x_{i}, y_{j}, x_{k}\right)$, contradicting the choice of $y_{j}$, which proves Claim 9.

Claim 10. Every $y_{j}$ is contained in a crossing quadruple.

Proof. Suppose there exists $y_{j}$ that is not contained in any crossing quadruple. By Claim 3, there exists $r$ such that $y_{j}=x_{r}$ and $N(y)-\{x\} \subseteq C\left[x_{r-1}, x_{r}, x_{r+1}\right]$. Note that $x_{r+2} \notin C\left[x_{r-1}, x_{r}, x_{r+1}\right]$ since $d(x) \geq 5$, as shown in Claim 9. Choose $y_{m}, y_{n}$ such that $N(y)-\{x\} \subseteq C\left[y_{m}, \bar{x}_{r+2}, y_{n}\right]$. Since $G$ is nonplanar, each of $C\left[x_{r-1}, \bar{x}_{r+2}, x_{r}\right)$ 
and $C\left(x_{r}, \bar{x}_{r+2}, x_{r+1}\right]$ contains one of $y_{m}$ and $y_{n}$. As a result, $\left(y_{m}, x_{r}, y_{n}, x_{r+2}\right)$ is a crossing quadruple. By Claim $5, x x_{r}$ is not contractible. It follows that there is a 4-separator $T$ containing both $x$ and $x_{r}$. Since $d(y) \geq 5$ (by Claim 9), Claim 6 implies that $T=\left\{x, x_{r}, z, z^{\prime}\right\}$ for some $z \in V(C)-\left\{x_{r}\right\}$ and $z^{\prime} \in V(B)$. Notice that $x_{r}$ is the only neighbor of $x$ in $C\left(y_{m}, x_{r}, y_{n}\right)$. This contradicts Claim 7 and thus Claim 10 is proved.

Claim 11. No edge of $C$ is contractible.

Proof. Suppose to the contrary that $f \in E(C)$ is a contractible edge of $G$. By Claim 2, $G$ has a crossing quadruple $\left(x_{i}, y_{j}, x_{k}, y_{l}\right)$. Let $H$ be the subgraph of $G$ formed by edges in $E(C) \cup\left\{x y, x x_{i}, x x_{k}, y y_{j}, y y_{l}\right\}$. Then $H$ is a subdivision of $K_{3,3}$. Since we assumed contracting any contractible edge leaves a planar graph, $G / f$ is planar. It follows that $H / f$ is no longer a subdivision of $K_{3,3}$. By symmetry, we assume $f=x_{i} y_{j}$.

If $C\left(x_{i}, \bar{y}_{j}, y_{l}\right)$ contains a neighbor $x_{m}$ of $x$, then $\left(H+x x_{m}\right) / f$ would still contain a subdivision of $K_{3,3}$, which is impossible. Hence $N(x)-\{y\} \subseteq C\left[x_{i}, y_{j}, y_{l}\right]$. This implies that $C\left(y_{j}, x_{i}, y_{l}\right)$ contains exactly one neighbor of $x$. However, by Claim $5, G$ has a 4 -separator $T$ that contains $\left\{x, x_{i}\right\}$ since $\left(y_{l}, x_{i}, y_{j}, x_{k}\right)$ is crossing. By Claim 6 and Claim 9, $T=\left\{x, x_{i}, z, z^{\prime}\right\}$ for some $z \in V(C)-\left\{x_{i}\right\}$ and $z^{\prime} \in V(B)$. Now, by Claim $7, C\left(y_{j}, x_{i}, y_{l}\right)$ contains at least two neighbors of $x$. This contradiction proves Claim 11.

Now we are ready to complete the proof of Lemma 3.1. We apply Lemma 2.3 to $G^{\prime}=G / x y$. By Claim $8, d_{G^{\prime}}(\overline{x y}) \geq 5$. Thus $E_{G^{\prime}}(v)$ contains a contractible edge $e^{\prime}$ of $G^{\prime}$ for some $v \in N_{G^{\prime}}(\overline{x y})$. By Lemma 2.5 and Claim 9, $e^{\prime}$ is contractible in $G$. However, by Claim 10 and Claim 5 , no edge of $E(\{x, y\})$ is contractible in $G$; by Claim 11, no edge of $C$ is contractible in $G$; and by Claim 1, cycle $C$ has no chords. Hence, $e^{\prime}$ is between $C$ and $B$. What this means is, for any crossing quadruple $\left(x_{i}, y_{j}, x_{k}, y_{l}\right)$, the graph formed by $E(C) \cup\left\{x y, x x_{i}, x x_{k}, y y_{j}, y y_{l}\right\}$ remains a subdivision of $K_{3,3}$ when $e^{\prime}$ is contracted from $G$. Thus $G / e^{\prime}$ is 4 -connected and nonplanar. The lemma is proved.

\section{A proof of the main theorem}

In this section we prove Theorem 1.2. Recall that a $(G, H)$-chain is a sequence $G_{0}, G_{1}, \ldots, G_{n}$ of 4 -connected graphs such that $G_{0}=G, G_{n}=H$, and every $G_{i}(i<n)$ has an edge $e_{i}$ such that $G_{i} / e_{i}=G_{i+1}$.

Proof of Theorem 1.2. Let $G$ be a 4-connected graph not in $\mathcal{C} \cup \mathcal{L}$. By Theorem 1.1, there exists a $\left(G, G_{n}\right)$-chain $G_{0}, G_{1}, \ldots, G_{n}$ such that $G_{n} \in \mathcal{C} \cup \mathcal{L}$. We choose such a chain as follows:

(1) if $G$ is planar, we choose the chain with as many terms as possible;

(2) if $G$ is not planar, we choose the chain with as many nonplanar terms as possible. 
If $G$ is planar, we need to show that $G_{n}=C_{6}^{2}$. Suppose otherwise. By applying Corollary 2.2 to $G_{n-1}$ and $e_{n-1}$ we obtain an edge $e_{n-1}^{\prime}$ of $G_{n-1}$ such that $G_{n}^{\prime}=G_{n-1} / e_{n-1}^{\prime}$ is 4-connected but $G_{n}^{\prime}$ does not belong to $\mathcal{C} \cup \mathcal{L}$, as it cannot be 4 -regular. Now, by Theorem 1.1 again, there exists a $\left(G_{n}^{\prime}, G_{m}^{\prime}\right)$-chain $G_{n}^{\prime}, G_{n+1}^{\prime}, \ldots, G_{m}^{\prime}$ with $G_{m}^{\prime} \in \mathcal{C} \cup \mathcal{L}$. It follows that $G_{0}, G_{1}, \ldots, G_{n-1}, G_{n}^{\prime}, G_{n+1}^{\prime}, \ldots, G_{m}^{\prime}$ is a chain contradicting the choice of (1), which proves the first part of the theorem.

If $G$ is nonplanar, let $G_{0}, G_{1}, \ldots, G_{k}$ be all the nonplanar terms. We need to show that $k=n$ and $G_{n}=K_{5}$. If $k<n$ then $G_{k} \notin \mathcal{C} \cup \mathcal{L}$ since no graph in $\mathcal{C} \cup \mathcal{L}$ has a contractible edge while $G_{k}$ has a contractible edge $e_{k}$. By Lemma 3.1, $G_{k}$ has a contractible edge $e_{k}^{\prime}$ such that $G_{k+1}^{\prime}=G_{k} / e_{k}^{\prime}$ is nonplanar. Like in the planar case, we can extend $G_{0}, G_{1}, \ldots, G_{k}, G_{k+1}^{\prime}$ to a chain that contradicts the choice of (2), which proves that $k=n$. If $G_{n} \neq K_{5}$, by applying Corollary 2.2 to $G_{n-1}$ and $e_{n-1}$ we obtain a contractible edge $e_{n-1}^{\prime}$ of $G_{n-1}$ such that $G_{n}^{\prime}=G_{n-1} / e_{n-1}^{\prime}$ is nonplanar and not in $\mathcal{C} \cup \mathcal{L}$. Consequently, by Lemma 3.1, $G_{n}^{\prime}$ has an edge $e_{n}^{\prime}$ such that $G_{n+1}^{\prime}=G_{n}^{\prime} / e_{n}^{\prime}$ is 4-connected and nonplanar. Now, once again, $G_{0}, G_{1}, \ldots, G_{n-1}, G_{n}^{\prime}, G_{n+1}^{\prime}$ can be extended into a chain. This contradicts the choice of (2), which completes our proof of the theorem.

\section{Acknowledgments}

We thank the anonymous referees for carefully reading the paper and for their helpful comments and suggestions. The first author is supported in part by NSF of China under grant 11401119. The second author is support in part by NSF under grant DMS-1500699.

\section{References}

[1] Kiyoshi Ando, Yoshimi Egawa, Contractible edges in a 4-connected graph with vertices of degree greater than four, Graphs Combin. 23 (2007) 99-115.

[2] Guoli Ding, Chanun Lewchalermvongs, John Maharry, Graphs with no $\bar{P}_{7}$-minor, Electron. J. Combin. 23 (2) (2016), \#P2.16.

[3] Max Fontet, Graphes 4-essentiels, C. R. Acad. Sci. Paris Sér. A-B 287 (5) (1978) 289-290.

[4] Max Fontet, Connectivité des graphes et automorphismes des cartes: propriétés et algorithmes, Thèse d'État, Université Paris VII, 1979.

[5] A.K. Kelmans, Graph expansion and reduction, in: Algebraic Methods in Graph Theory, vol. I, Szeged, Hungary, 1978, in: Colloq. Math. Soc. János Bolyai, vol. 25, North-Holland, Amsterdam, 1981, pp. 318-343.

[6] W. Mader, On k-critically n-connected graphs, in: J.A. Bondy, U.S.R. Murty (Eds.), Progress in Graph Theory, 1984, pp. 389-398.

[7] John Maharry, An excluded minor theorem for the octahedron, J. Graph Theory 31 (2) (1999) 95-100.

[8] John Maharry, A characterization of graphs with no cube minor, J. Combin. Theory Ser. B 80 (2) (2000) 179-201.

[9] John Maharry, An excluded minor theorem for the octahedron plus an edge, J. Graph Theory 57 (2) (2008) 124-130.

[10] Nicola Martinov, Uncontractable 4-connected graphs, J. Graph Theory 6 (3) (1982) 343-344.

[11] W.T. Tutte, How to draw a graph, Proc. Lond. Math. Soc. 13 (1963) 743-767.

[12] Nicholas Wormald, Classifying k-Connected Cubic Graphs, Lecture Notes in Mathematics, vol. 748, 1979, pp. 199-206. 